\title{
Temporal integration of vibrotactile patterns
}

\author{
JAMES C. CRAIG \\ Indiana University, Bloomington, Indiana
}

\begin{abstract}
Three experiments were conducted to measure the temporal integration of vibrotactile patterns presented to the fingertip. In Experiment 1, letters were divided in half and the time between the onsets of the first half of the letter and second half of the letter, stimulus onset asynchrony (SOA), was varied. The recognizability of the letters declined as the SOA was increased from 9 to $100 \mathrm{msec}$. In Experiment 2, the time between two patterns constituting a masking stimulus was varied and the stimulus effectiveness in interfering with letter recognition was determined. The amount of masking increased as the SOA increased from 9 to $50 \mathrm{msec}$. In Experiment 3, the SOA between a letter and its complement (the portions of the tactile array not activated by the letter) was varied. Increasing SOA from 9 to approximately $50 \mathrm{msec}$ led to increasingly accurate letter recognition. The results of the three experiments suggest that the skin is capable of complete temporal integration over a time period of less than $10 \mathrm{msec}$, and that the temporal integration function becomes asymptotic in 50 to $100 \mathrm{msec}$. The results also suggest that the onset of a vibrotactile pattern is critical for generating contours. The implications of the results for modes of generating tactile patterns and for temporal masking functions are discussed.
\end{abstract}

Several studies recently completed in this laboratory have employed the concept of "temporal integration" to explain results obtained in vibrotactile patternrecognition tasks (Craig, 1978, 1980, 1981). One study examined a number of modes of generating vibrotactile patterns, patterns created from letters of the alphabet. Pattern recognition was measured as a function of display time. For several of the modes, the elements making up the patterns were articulated both spatially and temporally. The results showed large differences among modes, with a general trend that longer display times produced better recognition; however, at the briefest display time, three of the modes showed some deviations from this trend, suggesting that the way in which the patterns were being processed was changing.

The three modes were: static, in which all elements of the pattern are turned on and off simultaneously; slit-scan, in which a portion of the pattern is exposed as though a narrow slit were being passed across the pattern; and discontinuous sequential, in which contiguous portions of the pattern are turned on and then off in sequence, as though the pattern were being drawn on the skin. In the latter two modes, patterns presented over time (and space) produced results similar to the static mode in which the elements were all present simultaneously. The similarity in the performance obtained with these modes declined as display time increased beyond the very briefest times.

This research was supported by Grant NS-09783 from the National Institutes of Health. The author wishes to thank Roger Rhodes for his assistance in conducting these experiments. The author's mailing address is: Department of Psychology, Indiana University, Bloomington, Indiana $\mathbf{4 7 4 0 5 .}$
These observations indicated that, at brief times, the nature of the temporal articulation of the patterns was unimportant. This implies that the elements were presented within the temporal integration time of the skin. Patterns presented in the slit-scan and discontinuous sequential modes were divided into six elements. At the briefest time, $26 \mathrm{msec}$, each element was presented for $4.3 \mathrm{msec}$. At the next longest time, $156 \mathrm{msec}$, each element was presented for $26 \mathrm{msec}$. Both the overall error rate and the nature of confusions among letters changed as display time was increased from 26 to $156 \mathrm{msec}$. At the briefest times, the overall error rates were similar for the three modes, as were the kinds of confusions among letters. Increasing the display time resulted in both a difference in overall error rates among modes and a decline in the correlations between modes based on the rank orderings of correct responses. These changes led us to speculate that the limit for complete temporal integration of patterns must be less than $156 \mathrm{msec}$ (Craig, 1981).

A second type of study that has provided information about temporal integration has measured the ability of subjects to recognize letters in the presence of forward and backward maskers. Generally, the results have shown more backward than forward masking (asymmetrical temporal masking functions) and have been interpreted in terms of current views of masking in the visual system. Specifically, two processes have been hypothesized to underlie temporal masking functions for visual stimuli, integration and interruption (Breitmeyer \& Ganz, 1976; Massaro, 1975; Turvey, 1973). Interruption is thought to operate in backward masking paradigms and to be responsible for producing greater amounts of backward than 
forward masking. Integration, if operating by itself, would, according to some authors, produce symmetrical temporal masking functions (Massaro, 1975). It has been speculated that the masking function obtained with vibrotactile patterns is the result of both interruption and the temporal integration of the target with the masker (Craig, 1978, 1980, 1981).

A number of studies have measured temporal summation and its effects on detection for relatively simple vibratory stimuli. The general finding, depending upon the size of the contactor and the frequency of vibration, is that the amplitude of vibration required for detection decreases rapidly as duration increases out to about $100 \mathrm{msec}$ and then more gradually out to several hundred milliseconds (Gescheider, 1976; Green, 1976; Verrillo, 1965). For suprathreshold stimuli, a similar picture emerges: The perceived intensity of a vibratory stimulus increases rapidly as duration increases out to $100 \mathrm{msec}$, and then continues to increase more gradually beyond that duration (Berglund, Berglund, \& Ekman, 1967). Although measures of summation provide an estimate of the time over which the skin can integrate energy, they do not provide a measure of the time over which the skin can integrate spatial patterns.

The present study measured the amount of interaction between elements of vibrotactile patterns as a function of the time between the onsets of these elements. The first aim of the study was to measure the temporal integration function for vibrotactile patterns directly. The second aim was to relate the temporal integration functions to previous studies of pattern recognition and temporal masking. The third aim was to determine which aspects of vibrotactile patterns were important for the generation of contours that could be temporally integrated.

\section{EXPERIMENT 1}

In the study of modes of generating vibrotactile patterns referred to before (Craig, 1981), the way in which the patterns were generated in the slit-scan and discontinuous sequential modes made it difficult to specify the time course of temporal integration. Each letter was divided into six parts, and it may be that subjects were able to obtain enough information to recognize the letter before all six elements were presented. To permit more exact specification of the time course of integration, the situation was simplified by dividing the letters into two parts. This procedure is similar to that used by Eriksen and Collins (1967) in their study of visual pattern perception.

\section{Method}

Subjects. All subjects were college students who were paid employees of the laboratory. They passed an initial screening test (Craig, 1980) and received several weeks of training before formal data collection began. Several groups of subjects were tested on the various measurements that Experiment 1 comprised.
Apparatus. The apparatus has been described previously (Craig, 1980). It consists of the tactile display of an Optacon interfaced with a PDP-11/34 computer. The tactile display is a 6 row $\times 24$ column array of pins that can be made to vibrate at $230 \mathrm{~Hz}$. The array measures of $1.1 \times 2.7 \mathrm{~cm}$ and fits against the distal portion of the subject's fingerpad.

Stimuli. The stimuli were five uppercase letters (B, E, M, R, W) shown previously to be relatively difficult to identify vibrotactually (Craig, 1979). The letters were divided in half along the vertical axis, along the horizontal axis, and along the two diagonal axes of the rectangular array on which they were displayed. On each trial, the two halves of the letter were presented sequentially. By dividing the letters along four axes and randomly selecting which half was presented first, a particular letter could be presented in eight different ways. An example of the patterns is shown in Figure 1.

Procedure. The subjects' task was to identify which of the five letters had been presented to the fingertip. The patterns occupied the top 18 rows of all 6 columns of the tactile array. The amplitude

$\begin{array}{llllll}0 & 0 & 0 & 0 & 0 & 0 \\ 0 & 0 & 0 & 0 & 0 & 0 \\ 0 & 0 & 0 & 0 & 0 & 0 \\ 0 & 0 & 0 & 0 & 0 & 0 \\ 0 & 0 & 0 & 0 & 0 & 0 \\ 0 & 0 & 0 & 0 & 0 & 0 \\ 0 & 0 & 0 & 0 & 0 & 0 \\ 0 & 0 & 0 & 0 & 0 & 0 \\ 0 & 0 & 0 & 0 & 0 & 0 \\ 0 & 0 & 0 & 0 & 0 & 0 \\ 0 & 0 & 0 & 0 & 0 & 0 \\ 0 & 0 & 0 & 0 & 0 & 0 \\ 0 & 0 & 0 & 0 & 0 & 0 \\ 0 & 0 & 0 & 0 & 0 & 0 \\ 0 & 0 & 0 & 0 & 0 & 0 \\ 0 & 0 & 0 & 0 & 0 & 0 \\ 0 & 0 & 0 & 0 & 0 & 0 \\ 0 & 0 & 0 & 0 & 0 & 0\end{array}$

$\begin{array}{llllll}0 & 0 & 0 & 0 & 0 & 0 \\ 0 & 0 & 0 & 0 & 0 & 0 \\ 0 & 0 & 0 & 0 & 0 & 0 \\ 0 & 0 & 0 & 0 & 0 & 0 \\ 0 & 0 & 0 & 0 & 0 & 0 \\ 0 & 0 & 0 & 0 & 0 & 0 \\ 0 & 0 & 0 & 0 & 0 & 0 \\ 0 & 0 & 0 & 0 & 0 & 0 \\ 0 & 0 & 0 & 0 & 0 & 0 \\ 0 & 0 & 0 & 0 & 0 & 0 \\ 0 & 0 & 0 & 0 & 0 & 0 \\ 0 & 0 & 0 & 0 & 0 & 0 \\ 0 & 0 & 0 & 0 & 0 & 0 \\ 0 & 0 & 0 & 0 & 0 & 0 \\ 0 & 0 & 0 & 0 & 0 & 0 \\ 0 & 0 & 0 & 0 & 0 & 0 \\ 0 & 0 & 0 & 0 & 0 & 0 \\ 0 & 0 & 0 & 0 & 0 & 0 \\ 0 & 0 & 0 & 0 & 0 & 0 \\ 0 & 0 & 0 & 0 & 0 & 0 \\ 0 & 0 & 0 & 0 & 0 & 0 \\ 0 & 0 & 0 & 0 & 0 & 0 \\ 0 & 0 & 0 & 0 & 0 & 0 \\ 0 & 0 & 0 & 0 & 0 & 0\end{array}$

Figure 1. A representation of the letter $B$ as it appeared on the tactile display with a 0 -msec SOA, and the way it appeared when divided in half along the positive diagonal in Experiment 1. 
of vibration was set at a comfortable level, $35 \mathrm{~V}$ to each pin, and remained unchanged for all three experiments.

The subjects placed their left index fingers on the array. Throughout the testing sessions, the subjects wore earphones through which white noise was presented to reduce auditory cues from the tactile display. Trials were self-paced. The subject pressed a key and received a tactile cue stimulus, followed $1 \mathrm{sec}$ later by the two letter halves. The subjects responded by pressing one of five response keys. No feedback was given.

To examine the effect of varying amounts of temporal overlap between patterns while examining similar stimulus onset asynchronies (SOAs), the pattern halves were presented for three different durations: 4,13 , or $26 \mathrm{msec}$. With the $4-\mathrm{msec}$ halves, the SOA was set at $0 \mathrm{msec}$, that is, the whole letter presented for $4 \mathrm{msec}$, or at $9,13,17,22,30,56,104$, or $200 \mathrm{msec}$. With the $13-\mathrm{msec}$ halves, the SOA was set at $0,4,9,17,30,56,154$, or $254 \mathrm{msec}$. With the $26-\mathrm{msec}$ halves, the SOA was set at $0,4,13,30$, $56,104,204$, or $304 \mathrm{msec}$. Also, a half-letter condition was included for all three durations to determine how well subjects could identify the letters using the information present in only half of the letter.

The subjects were tested in 40-trial blocks. The same SOA was tested throughout the block, and each SOA was tested in random order during a single experimental session. Testing was completed with the 26-msec patterns before it was begun with the 13-msec patterns. The 4-msec patterns were tested, after data collection was completed with the $13-\mathrm{msec}$ patterns, with a different group of subjects.

After data collection had been completed with all three durations, an error was discovered in the program controlling the timing of the two halves of the patterns. Specifically, in cases in which the two halves of the letter overlapped temporally, the second half of the letter was turning off for one cycie $(4 \mathrm{msec})$ at the offset of the first half of the letter, producing a gap that might have affected the results for the briefer SOAs in the 26- and 13-msec conditions. Therefore, the 26-msec halves were retested with one group of three subjects, and the 13-msec letter halves were retested with a second group of three subjects. The retesting produced no evidence that the gap affected the data already collected.

\section{Results and Discussion}

Each point in the 26- and $13-\mathrm{msec}$ functions in Figure 2 is the result of 1,120 trials, 280 trials from each of four subjects. The 4-msec data were collected from three subjects and represent 900 trials, 300 trials per subject. The standard error of the mean, computed for each point by averaging across blocks, ranged from $2 \%$ to $3 \%$ and did not vary significantly as a function of SOA or stimulus duration. (For the results reported in all three experiments, the standard errors of the mean did not exceed $4 \%$ and did not vary systematically as a function of experimental conditions.) The results show that the majority of temporal integration takes place within 50 to $100 \mathrm{msec}$ of the onset of the first pattern, a result similar to temporal summation for simple sinusoids (Berglund et al., 1967; Gescheider, 1976; Green, 1976; Verrillo, 1965). The results are also similar, although not identical, to Eriksen and Collins's (1967) visual results, in which the major decline in performance occurred when the interstimulus interval (ISI) was increased from 0 to $100 \mathrm{msec}$, with some decrease occurring when the ISI was increased from 100 to $300 \mathrm{msec}$.

The 4-msec function shows that performance declines somewhat as the time between the onsets of the two halves of the pattern is increased from 0 to $26 \mathrm{msec}$. This decline in performance over that temporal interval is not entirely in accordance with the speculation, arising from the study of modes of pattern generation (Craig, 1981), that patterns with elements coming on sequentially over time show complete temporal integration if the total time is brief enough. In the previous work, the last element in the pattern came on $22 \mathrm{msec}$ after the onset of the first element. However, in that case, there were four other elements of the pattern being turned on between the first and last elements. It is possible, indeed likely, that subjects were accumulating information about the letter continuously, and that all six elements were not required to identify the letter, thus reducing the time over which integration was necessary.

The data in Figure 2 also show that there is little, if any, temporal integration beyond about 50 to $100 \mathrm{msec}$. This result is consistent with the previous speculation that patterns taking longer than 100 to $150 \mathrm{msec}$ to be articulated (i.e., the time between the onset of the first and last elements is greater than $100-150 \mathrm{msec}$ ) show no temporal integration of their features, and so must not be processed in parallel.

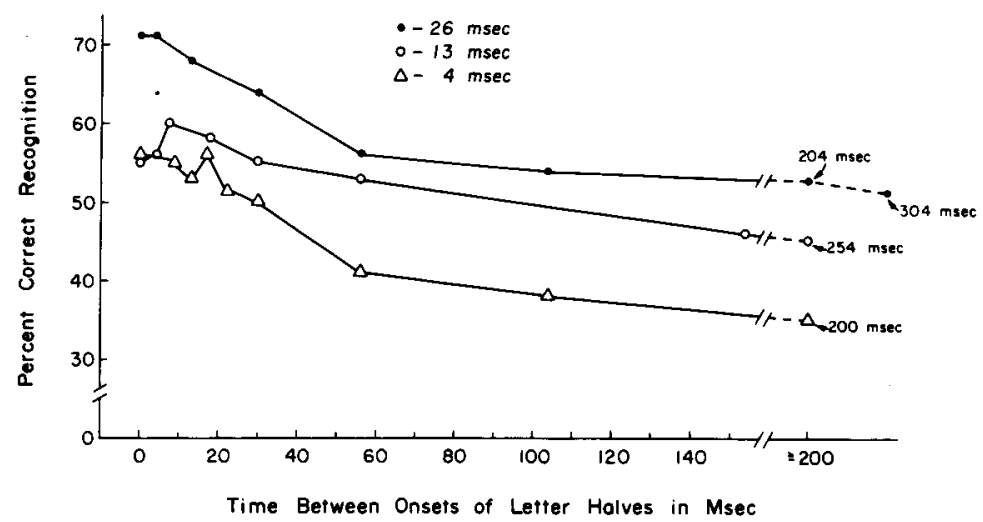

Figure 2. Percentage correct letter recognition as a function of the time between the onsets of the two halves constituting the letter. The results from three durations of letter halves are shown. 
In this type of task, the performance level achieved when only half the letter is presented forms an important baseline condition. If patterns can be identified readily when only half of the pattern is presented, then it will not be possible to see any effect of SOA between pattern halves on recognition. Also, if performance levels at the longer SOAs never approach those achieved when only a single letter half is presented, then either the SOAs are not long enough to yield asymptotic performance or, perhaps more likely, subjects are able to encode pattern features in a way that does not vary with SOA. That is, subjects might be able to encode verbally some features from the first letter half such that the presentation of the second half improves their response accuracy. In preliminary work using all 26 letters of the alphabet, the level of performance even at very long SOAs remained well above half-letter recognition, indicating that subjects were able to encode features verbally. In the present work, however, the asymptotic performance levels at the longest SOAs averaged only $6 \%$ above the half-letter performance, a result that suggests that the integration seen in Figure 2 is not the result of verbal encoding strategies.

A comparison of the 4- and 26-msec functions shows that the amount of temporal overlap of the two halves of the patterns has very little effect on the form of the temporal integration function. For an SOA of from 0 to $26 \mathrm{msec}$, the two halves of the 26msec patterns overlap, whereas beyond an SOA of $0 \mathrm{msec}$ there is no overlap of the two halves for the 4-msec patterns. The strong implication of this surprising result is that the contours of a vibrotactile pattern are generated by the onset of the pattern, and that generation of the contours is independent of the continuation of the pattern.

Because these results suggest the importance of onset and the relative unimportance of the amount of temporal overlap between pattern halves, another group of four subjects was tested under conditions that might serve to highlight these observations. Specifically, three stimulus durations, 4,26 , and $52 \mathrm{msec}$, and four SOAs, 0, 9, 26, and $56 \mathrm{msec}$, were tested. To see the effect of onset more clearly, the data were plotted to show changes in percent correct from singleletter performance (0-msec SOA) as a function of SOA. Each point represents the mean of 1,320 trials, 330 from each of the four subjects.

The results show that, under conditions with quite different amounts of temporal overlap, the amount of temporal integration, as reflected in the change in percentage correct, is similar. For example, at 9-msec SOA, the three functions produce the same results, no decline in performance from a 0-msec condition; yet, for the 4-msec patterns there is a 5-msec ISI, whereas for both other stimulus durations the two halves overlap considerably. At 26-msec SOA, the 4and $52-\mathrm{msec}$ patterns produce nearly the same results, even though for the briefer pattern there is a $22-\mathrm{msec}$ ISI and for the longer pattern the two halves overlap by $26 \mathrm{msec}$. At $56-\mathrm{msec} \mathrm{SOA}$, the $26-$ and $52-\mathrm{msec}$ patterns produce nearly the same results with quite different ISIs. Even the point that deviates most widely from the trend of the results, the 4-msec pattern at 56-msec SOA, is opposite in direction to what would be predicted if ISI were important. At that point, the 4-msec halves are separated by $52 \mathrm{msec}$ and show a smaller decline in performance than the other two patterns with briefer ISIs.

The results in Figure 3 also show that there is no decline in performance, that is, complete temporal integration, out to $9 \mathrm{msec}$. The data in Figure 2 also show good temporal integration at the very briefest SOAs.

One of the reasons for making the present measurements of temporal integration was to help explain results obtained with various modes of generating vibrotactile patterns on the fingertip. The results of the previous mode measurements on the fingertip (Craig, 1981) were in disagreement with some studies that examined vibrotactile pattern generation on larger areas of skin (i.e., the back). Specifically, Loomis (1974) found that subjects recognized larger patterns presented on the back more readily when the patterns were elaborated sequentially over time as compared with the condition in which the elements were turned on simultaneously (i.e., static presentation). The reverse was found for patterns presented to the fingertip, leading to the speculation that the smaller patterns on the fingertip were processed in parallel, whereas the larger patterns on the back were processed serially (Craig, 1981). In Experiment 1, the decline in performance as time between the two halves of the letter increased, even though the greater times would presumably permit serial processing, supports the conclusion that parallel processing is the "preferred" mode for the fingertip. The same result also

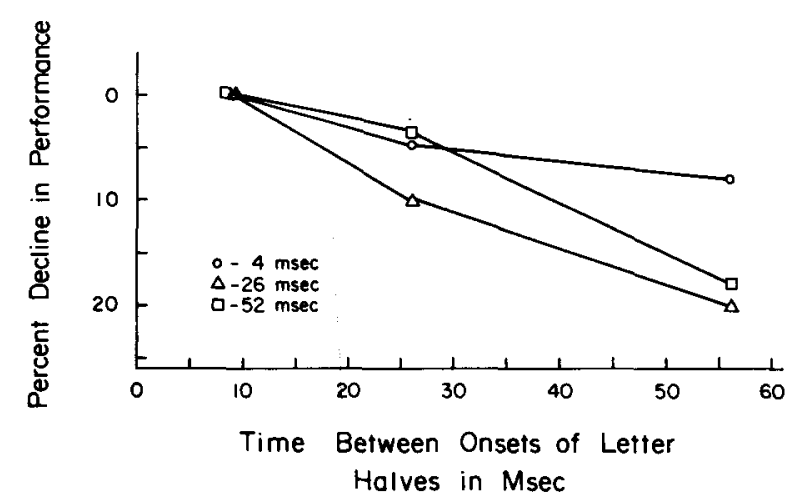

Figure 3. Percentage decline in performance from a 0-msec SOA condition (complete letter) as a function of the time between the onsets of the two halves constituting the letter. The results from three durations of letter halves are shown. 
suggests that if patterns are divided into parts and presented to larger areas of skin, where serial processing may be superior, performance might actually improve rather than decline as the time between parts of the patterns increases, at least over relatively brief temporal intervals. Because in previous studies display time has been confounded with SOA, so that improvements in performance may have been due to either or both of these factors, additional measurements would be necessary to separate these factors.

\section{EXPERIMENT 2}

It has recently been demonstrated that a pattern mask, generated by presenting parts of letters, interferes more in letter recognition than does an energy mask, generated by turning on all the pins in the array covering the letter (Craig, 1982). Taking advantage of this difference in effectiveness, Experiment 2 measured the time course of an energy masker becoming a pattern masker. An energy masker was presented immediately after a target letter, and its effectiveness as a backward masker was determined. Then the energy masker was divided into two pattern maskers, separated by a temporal interval. One of the two pattern maskers was the "complement" of the other such that when the SOA between the two pattern maskers was $0 \mathrm{msec}$, they would form an energy masker. The temporal separation required before the two pattern maskers began to produce more interference and the separation beyond which no increase in masking occurred was taken as the range of temporal integration.

It was expected that this task would produce a function similar to those seen in Experiment 1. If there is any increase in the amount of masking, it is assumed that it is the result of the emergence of contours in the first pattern mask interfering with the contours of the target letters. As in Experiment 1, the contours of the first pattern should last for some brief period of time and be "connected with," integrated with, the contours of the second pattern. To the extent that this happens, the masker remains an energy masker and letter recognition should remain relatively good.

\section{Method}

Subjects. Four subjects were selected and trained as in Experiment 1 .

Apparatus. The apparatus used in Experiment 2 was the same as that used in Experiment 1.

Procedure. The subjects were required to identify which one of 26 uppercase letters of the alphabet had been presented. The subjects were presented the target letters either singly or with the target letter, followed, $4 \mathrm{msec}$ after its offset, by a masking stimulus. The maskers were of two types, either an energy masker, created by turning on all the pins in the top 18 rows and 6 columns of the array, or two pattern maskers. The first of the two pattern maskers was created, as in a previous study (Craig, 1982), by dividing the top 18 rows and 6 columns into six equal-sized parts. Each part contained a portion of a letter, such as a vertical, horizontal, or curved line. The second pattern masker was created by turning on all the pins in the top 18 rows and all 6 columns of the display that were not activated by the first pattern masker; that is, the second masker was the complement of the first. Examples of the stimuli used may be seen in Figure 4. Presenting the two pattern maskers with a 0 -msec SOA was equivalent to presenting the energy masker. Put another way, the energy masker was "pulled apart" to create two pattern maskers.

In the first set of measurements, 26 different pairs of pattern maskers were used. The mean number of pins in the first pattern was 41 ; the mean number in the second pattern was 67 . Both maskers were presented for $13 \mathrm{msec}$. Twenty-six different masker pairs were used because, as observed by Craig (1982), one might expect less masking from repeated use of a single pattern masker than from the less frequent use of 26 different pattern maskers. The intent was to produce significant amounts of masking, even

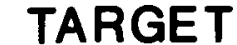

$\begin{array}{llllll}0 & 0 & 0 & 0 & 0 & 0 \\ 0 & 0 & 0 & 0 & 0 \\ 0 & 0 & 0 & 0 & 0 & 0 \\ 0 & 0 & 0 & 0 & 0 & 0 \\ 0 & 0 & 0 & 0 & 0 & 0 \\ 0 & 0 & 0 & 0 & 0 & 0 \\ 0 & 0 & 0 & 0 & 0 & 0 \\ 0 & 0 & 0 & 0 & 0 & 0 \\ 0 & 0 & 0 & 0 & 0 & 0 \\ 0 & 0 & 0 & 0 & 0 & 0 \\ 0 & 0 & 0 & 0 & 0 & 0 \\ 0 & 0 & 0 & 0 & 0 & 0 \\ 0 & 0 & 0 & 0 & 0 & 0 \\ 0 & 0 & 0 & 0 & 0 & 0 \\ 0 & 0 & 0 & 0 & 0 & 0 \\ 0 & 0 & 0 & 0 & 0 & 0 \\ 0 & 0 & 0 & 0 & 0 & 0 \\ 0 & 0 & 0 & 0 & 0 & 0 \\ 0 & 0 & 0 & 0 & 0 & 0 \\ 0 & 0 & 0 & 0 & 0 & 0 \\ 0 & 0 & 0 & 0 & 0 & 0\end{array}$

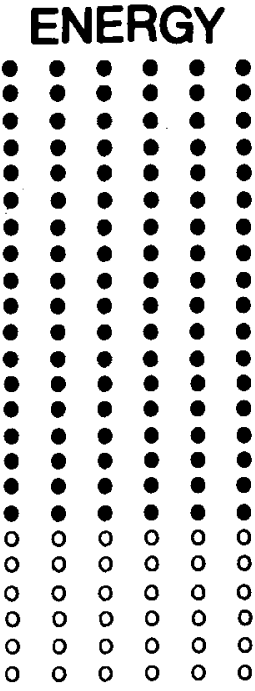

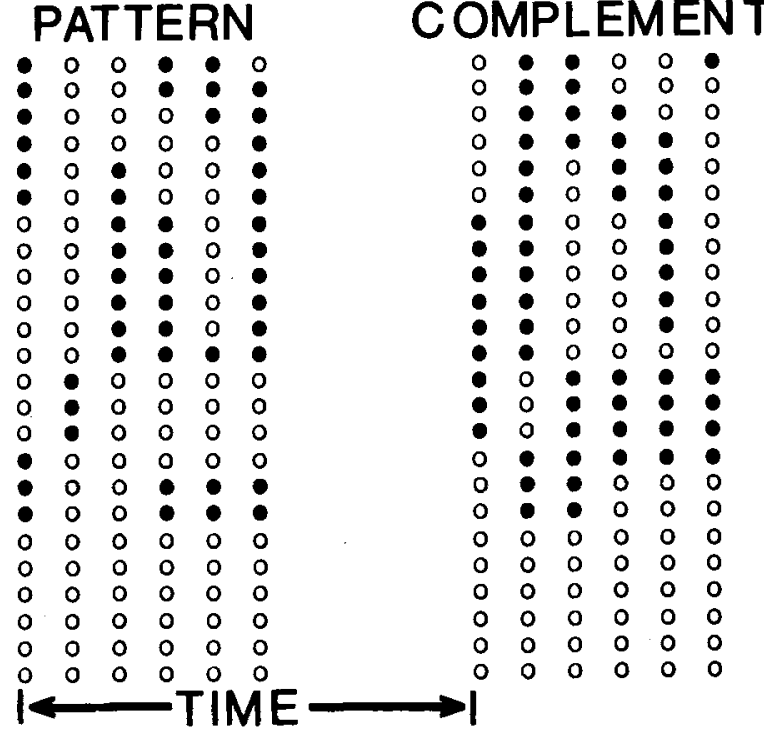

Figure 4. Representations of a target letter, $\mathbf{K}$, an energy masker, a pattern masker, and the pattern masker's complement; Experiment 2. 
though the first masker was briefer and therefore less intense than the target. The target was presented for $26 \mathrm{msec}$.

In a second condition, both the maskers and targets were presented for $26 \mathrm{msec}$, and the same two pattern maskers were used throughout. The first pattern masker was composed of 50 pins, and the second of 58 pins.

In both conditions, the energy masker and the first pattern masker were presented $4 \mathrm{msec}$ after the offset of the target letter. The second pattern masker was the complement of the first and followed it at various SOAs. The energy masker in the first condition was set at the same duration as the pattern maskers, $13 \mathrm{msec}$. The SOAs between the two pattern maskers were $4,9,17,43,90$, and $190 \mathrm{msec}$. In this condition, the energy masker was also set at the same duration as the pattern masker, $26 \mathrm{msec}$. The SOAs between the two pattern maskers were 4, 13, 30, 56, 104, and 204 msec. In addition, the amount of interference produced by a single pattern masker (not followed by its complement) was also determined.

The reason for using a complement of the pattern masker, rather than simply selecting two pattern maskers at random, was to allow the two maskers to overlap temporally. If they had also overlapped spatially, as would have been the case with two pattern maskers chosen at random, then the total number of pins activated and the duration of activation of the pins would be changing as a function of SOA. Such changes would make interpretation of the data difficult.

The testing procedure was similar to that used in Experiment 1. Subjects were tested in 40-trial blocks, with each block containing a different masking condition. Trial-by-trial feedback was provided. The 26-msec maskers were tested before the 13-msec maskers.

The programming error described in Experiment 1 also affected the presentation of the pattern maskers in Experiment 2. To see if this had any significant effect on the results, additional data were collected with both the 13- and 26-msec maskers at the briefer SOAs.

\section{Results and Discussion}

The results with the 13-msec masker are presented in Figure 5. Each point represents 280 trials from each of four subjects. The results are presented as the change in percentage correct, using a no-mask condition as a baseline, as a function of the time between the onsets of the pattern mask and its complement. The point at $0 \mathrm{msec}$ represents the level of performance with an energy masker; that is, all the pins constituting

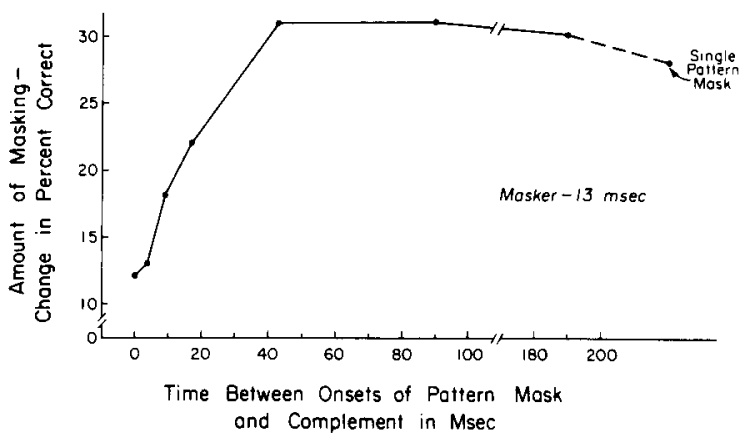

Figure 5. The amount of masking (decline in performance from the condition in which no masking stimulus was presented) produced by 13-msec pattern masker and complement as a function of the time between the onset of the pattern masker and complement. The data point at 0 -msec SOA represents performance with an energy masker. The data point marked "Single Pattern Mask" represents performance with a single pattern masker presented without its complement.

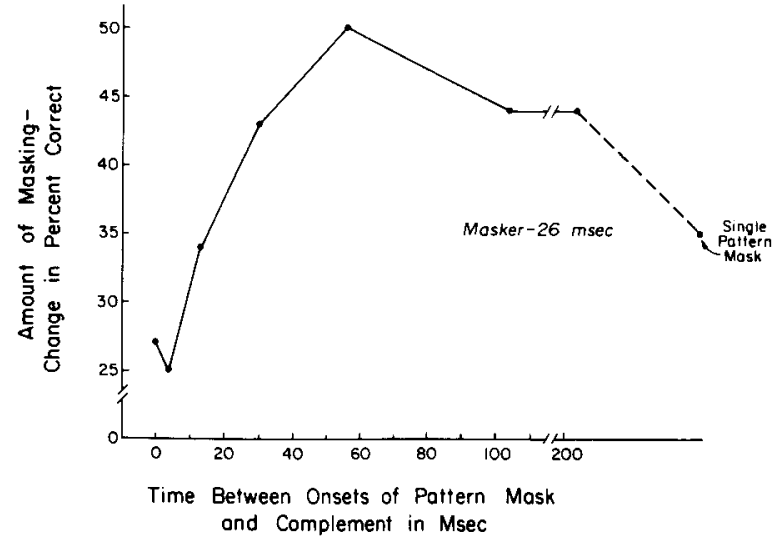

Figure 6. Results with a 26-msec masker and complement plotted as in Figure 5.

the masker are turned on and off simultaneously. Comparing that point with the point marked "single pattern masker" shows the effect of structuring the masker and replicates previous results (Craig, 1982) in that more masking is produced by a pattern than by an energy masker.

The main effect of increasing SOA is to increase the amount of masking, presumably because the contours of the first pattern mask are emerging from those of its complements. The second pattern mask appears to have little or no direct effect on the target letter inasmuch as the percentages of correct responses with the two patterns separated by 43,90 , or $190 \mathrm{msec}$ are essentially equal to one another and to recognition with the single pattern mask. Such may not be the case with the longer duration, and thus more intense, 26-msec masker.

The results with the 26-msec maskers are shown in Figure 6. Each point represents 280 trials from each of four subjects. Here, as in Figure 5 , the amount of masking increases out to $50 \mathrm{msec}$; however, beyond $50 \mathrm{msec}$, the amount of masking declines. It appears that the second masker, the complement, is, in this case, exerting some influence on the target at briefer SOAs. Evidence that this is happening is, first, that increasing the SOA from 56 to 104 (moving the complement further away from the target) results in a decline in masking and, second, that interference with the two maskers is greater than that produced by the single pattern masker. Previous measures of backward masking (using a single masker) have shown effects out to several hundred milliseconds, making the present explanation plausible. The results in Figure 6 do not show a decline in masking when the time between the second masker and the target is increased by $100 \mathrm{msec}$ (SOA increased from 104 to $204 \mathrm{msec}$ ). However, this result has also been seen with single backward maskers (Craig, 1980), that is, little or no change in performance observed when the time between target and masker changed by as much 
as $100 \mathrm{msec}$, when such a change is made at relatively long times between the masker and the target.

The additional data collected to see if the programming error had affected the results showed no major deviations from the results already collected. These additional data, plus the results for SOAs out to $56 \mathrm{msec}$ from Figures 5 and 6, were replotted to show changes in percentage correct from the energy masker condition (0-msec SOA, rather than a no-mask condition as in Figures 5 and 6 ). These results are shown in Figure 7. Each point from these additional data for the 13-msec condition represents the mean of 240 trials for each of three subjects; each point for the 26-msec condition represents the mean of 240 trials for each of four subjects. A linear regression was performed on the data and produced the straight line shown (slope $=.45, r=.96$ ). It appears that a single function can be fit to sets of data that differ in the amount of temporal overlap as long as the results are plotted in terms of SOA. Linear regressions performed on the 26- and 13-msec data separately produced slopes very similar to those obtained in the combined data, .47 and .44 , slopes that did not differ significantly from one another. The two functions produced the same fit to the data as the single function did.

The SOA over which there was complete temporal integration appears to be slightly briefer than it was in Experiment 1, 4 vs. 9 msec. Also, the SOAs at which there ceases to be an increase in the amount of masking is shorter, $50 \mathrm{msec}$, than would be predicted based on the results from the first experiment. The differences at the briefer SOAs are small, and also one could argue that the major portion of temporal integration is nearly complete at $50 \mathrm{msec}$ in Experiment 1 . In view of the variability of the results and the differences in procedure in Experiments 1 and 2, it was felt that additional measurements would be needed to determine if these differences were significant.

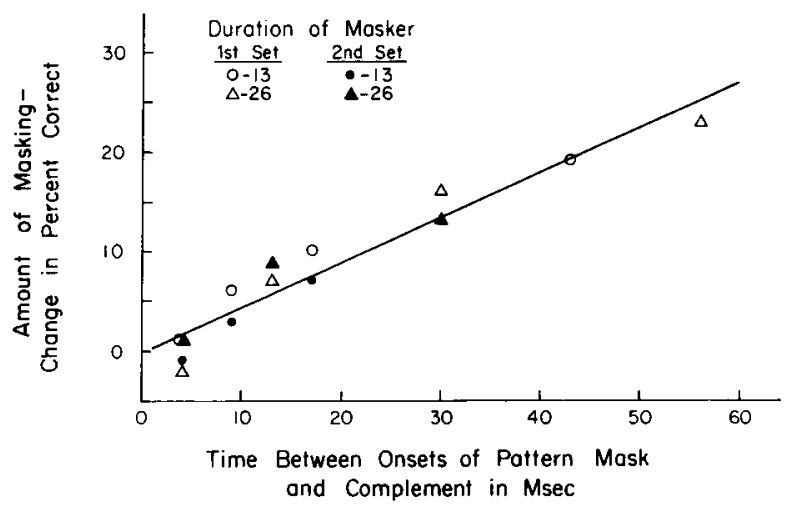

Figure 7. Amount of masking (decline in percentage correct) as a function of the time between a pattern mask and its complement. The decline in percentage correct is measured from the energy mask condition rather than a no-mask condition. The data from Figures 5 and 6 (Set 1) as well as additional data (Set 2) are presented.

\section{EXPERIMENT 3}

In Experiment 1, subjects were required to use the information from the two halves of the pattern to identify it. In Experiment 2, the effectiveness of a pattern masker was determined as it "emerged from" its complement. Experiment 3 used a paradigm similar to that of Experiment 2, in that a pattern emerges from its complement, but in this case subjects were required to identify the pattern. This procedure is more akin to the temporal masking studies that we have conducted previously, using 26 letters of the alphabet as targets. The situation was simplified by presenting subjects with one of only two possible patterns and their complements.

\section{Method}

Subjects. The subjects were selected and trained as in Experiment 1 .

Apparatus. The apparatus was the same as that used in Experiment 1.

Procedure. The subjects were required to indicate which one of two uppercase letters, $X$ or $O$, had been presented to their fingertips. Figure 8 shows a representation of the two patterns and the corresponding complements that were presented with them.

The target letters were presented for $26 \mathrm{msec}$, as were their complements. The SOAs between the target and its complement were set at $4,9,13,30,56$, and $104 \mathrm{msec}$, with the complement always presented after the target. If a 0-msec condition had been used, the pattern would consist of all 18 rows and 6 columns of the tactile display being turned on simultaneously. The two target patterns, $X$ and $O$, would then be indistinguishable. When presented without their complements, the two patterns could be identified with nearly perfect accuracy, $99.8 \%$ correct.

Using the same stimuli, a second set of measurements was made using four additional subjects. In these measurements, the complements either followed, as in the first set of measurements, or preceded the target letter. The same SOAs were tested. Alternate experimental sessions tested the forward or backward complement conditions.

The subjects were tested in 50-trial blocks. Each block tested a different SOA. Trial-by-trial feedback was provided.

\section{Results and Discussion}

The results from the first set of measurements are shown in Figure 9. Each point represents the mean of 300 trials from each of three subjects.

The main result of Experiment 3 is that the time course over which performance improves is similar to that seen in Experiments 1 and 2, approximately 30 to $50 \mathrm{msec}$. The results also show that as brief a time as $9 \mathrm{msec}$ between the onset of the stimuli is sufficient for the subjects to begin identifying the patterns. With a 9-msec SOA, the pattern and its complement overlap by $17 \mathrm{msec}$; that is, for 17 of $26 \mathrm{msec}$, the pattern and its complement are on simultaneously and provide no information about the pattern. However, at a 9-msec SOA, the complement of the pattern is also present for $9 \mathrm{msec}$; and, because the complement contains information about the nature of the pattern, there is, in effect, an 18-msec presentation of information that subjects might use to recognize the patterns. Subsequent measurements 


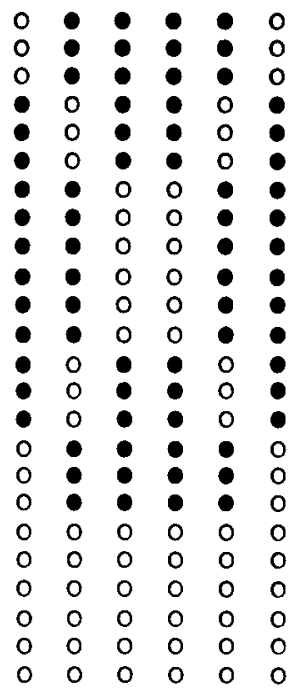

$\begin{array}{llllll}0 & 0 & 0 & 0 & 0 & 0 \\ 0 & 0 & 0 & 0 & 0 & 0 \\ 0 & 0 & 0 & 0 & 0 & 0 \\ 0 & 0 & 0 & 0 & 0 & 0 \\ 0 & 0 & 0 & 0 & 0 & 0 \\ 0 & 0 & 0 & 0 & 0 & 0 \\ 0 & 0 & 0 & 0 & 0 & 0 \\ 0 & 0 & 0 & 0 & 0 & 0 \\ 0 & 0 & 0 & 0 & 0 & 0 \\ 0 & 0 & 0 & 0 & 0 & 0 \\ 0 & 0 & 0 & 0 & 0 & 0 \\ 0 & 0 & 0 & 0 & 0 & 0 \\ 0 & 0 & 0 & 0 & 0 & 0 \\ 0 & 0 & 0 & 0 & 0 & 0 \\ 0 & 0 & 0 & 0 & 0 & 0 \\ 0 & 0 & 0 & 0 & 0 & 0 \\ 0 & 0 & 0 & 0 & 0 & 0 \\ 0 & 0 & 0 & 0 & 0 & 0 \\ 0 & 0 & 0 & 0 & 0 & 0 \\ 0 & 0 & 0 & 0 & 0 & 0 \\ 0 & 0 & 0 & 0 & 0 & 0 \\ 0 & 0 & 0 & 0 & 0 & 0 \\ 0 & 0 & 0 & 0 & 0 & 0 \\ 0 & 0 & 0 & 0 & 0 & 0\end{array}$

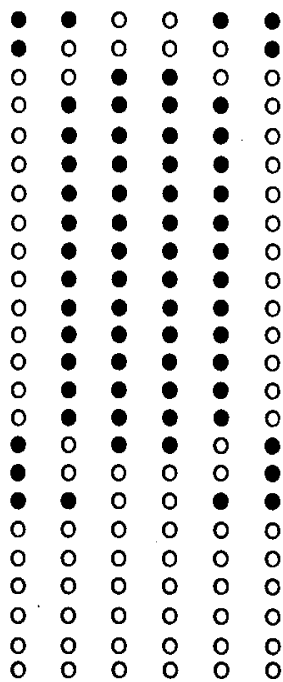

Figure 8. Representation of stimuli, letters $X$ and $O$ and their complements; Experiment 3.

showed that the complements were slightly more difficult to identify than the letters, $90.5 \%$ correct, suggesting that subjects were getting the major portion of their information from the letter rather than the complement.

The relationship between temporal masking functions and temporal integration is complicated. If, as indicated before, integration underlies at least part of the temporal masking function, then the variables that are known to affect masking should also affect temporal integration. For example, increasing the intensity of the masker increases the amount of both forward and backward tactile masking (Craig, 1982), and additional data collected in our laboratory show that the time over which masking occurs also increases with increasing intensity of the masker. Thus, varying the intensity of the complement in Experiment 3 might well change the temporal integration function.

Another manipulation known to change the amount of masking is the temporal position of the masking stimulus; that is, backward maskers produce more interference than forward maskers. To the extent that subjects are extracting most of the information on which they base their decisions from the letter rather than from the complement, the results in Figure 9 should resemble those found in a backward masking paradigm. Reversing the order of presentation of the pattern and complement should, in effect, produce a forward masking condition.

The results of these measurements are shown in Figure 10. Each point represents the mean of 800 trials, 200 from each of four subjects. The positive SOAs, complement following letter, produce a function similar to that seen in Figure 9. The slightly lower level of performance seen in the backward function in Figure 10, particularly at the longer SOAs, is most likely due to different subjects' being tested.

The forward and backward functions shown in Figure 10 reflect different levels of performance and are similar to results we have obtained in measures of forward and backward masking (Craig, 1976, 1980, 1981). However, the present results suggest that in this task the SOA at which performance levels off,

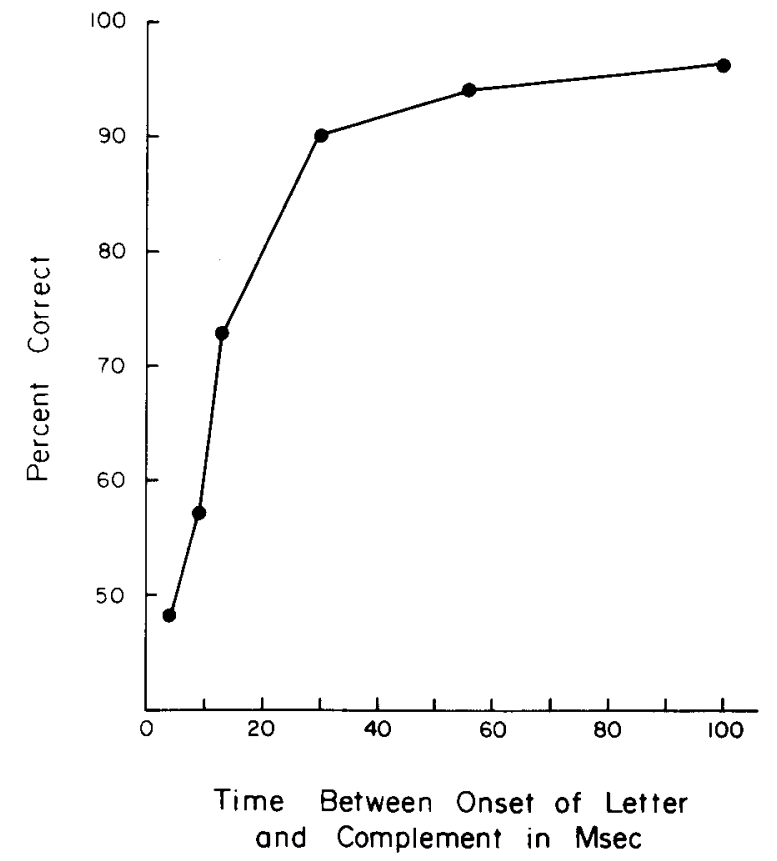

Figure 9. Percentage correct letter recognition as a function of the time between the onset of the letter and its complement. Complement follows letter. 


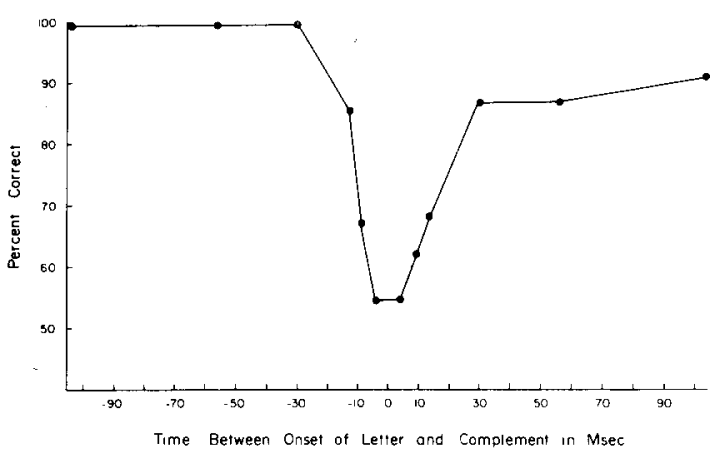

Figure 10. Percentage correct letter recognition as a function of the time between the onset of the letter and its complement. Positive temporal values represent conditions in which letter preceded complement; negative temporal values represent conditions in which complement preceded letter.

$30 \mathrm{msec}$, is the same for the forward and backward functions. It might be argued that these results are consistent with models of masking that suggest that integration operating alone produces symmetrical masking functions, and that it is the addition of interruption that produces characteristic asymmetrical functions. According to this view, the shallower slope of the function from +4 to $+30 \mathrm{msec}$ and the lower level of performance, particularly at the longer SOAs, in the backward function is the result of interruption. Interruption is thought to operate, at least for visual stimuli, over a longer time course than integration (Massaro, 1975).

However, it should be noted that visual results similar to those seen in Figure 10 have not necessarily been interpreted as supporting interruptive mechanisms (Felsten \& Wasserman, 1980). Also, integration may be affected by the difference in intensity between the target and its complement. The complements were composed of a greater number of pins than the targets; thus, in the forward function, a stronger complement is followed by a weaker target, whereas the reverse is true for the backward function. Additional measurements in which the targets and complements are equated for intensity should clarify the role of this intensity imbalance. Additional work should also manipulate the overall level of performance by increasing the number of patterns presented, and examine longer SOAs in both the forward and backward functions. The manipulations will be useful in overcoming the potential problem created by the nearly perfect levels of performance at the longer SOAs in the forward condition and in observing the full time course of these functions.

\section{GENERAL DISCUSSION}

The results from the three experiments indicate that the tactile system is capable of integrating information from spatially contiguous areas over brief temporal separations. It appears that the skin is rather sharply tuned temporally, in that when the time between the onsets of elements is as brief as $9 \mathrm{msec}$ the elements are not completely integrated (Figures 9 and 10), and that integration ceases within 50 to 100 msec.

Although previous studies of vibrotactile temporal summation have produced functions with longer time courses than those seen here (Berglund et al., 1967; Gescheider, 1976; Green, 1976; Verrillo, 1965), both temporal summation and pattern integration do show the major portion of the change in sensitivity to occur in the first 50 to $100 \mathrm{msec}$. This observation raises the question of whether or not the present results might be accounted for in terms of changes in the perceived intensity of the patterns. The results from Experiment 1 may lend themselves to such an explanation. It has been shown previously that reducing the intensity of statically presented vibrotactile patterns reduces their recognizability (Craig, 1980). If, as the time between the two halves of the pattern increases, the perceived intensity of the overall pattern decreases, a reduction in recognizability might result.

However, it is more difficult to extend the intensity explanation to the results of Experiments 2 and 3. In Experiment 2, it would have to be assumed that as the time between the two maskers increased, the intensity of the first masker decreased, a result that should lead to less rather than more masking. In Experiment 3, the intensity explanation would hold that, as the SOA increased, the intensity of the target and hence the level of performance should decrease, a conjecture clearly at odds with the results.

Rather than its being intensity that is integrated, it appears that it is information about tactile contours that is being integrated. The view that emerges from these experiments is that the onset of a cutaneous stimulus generates a contour, the representation of which begins to fade almost immediately (within $10 \mathrm{msec}$ ), and reaches asymptotic levels in about $50 \mathrm{msec}$. If the onset of a second stimulus occurs within that 50-msec interval, the contours of the second stimulus may be "added" to the contours already present. In the case of sequentially presented letter halves, information may be extracted from the combined representation of the two halves to see if it matches the stored representation of a complete pattern. As SOA increases, the representation of the first half fades and results in an increasingly imperfect representation of the complete pattern.

In Experiment 2, if the onsets of the two pattern maskers occurred close in time, an energy masker was generated. As SOA increased, the representation of the first pattern became less precise, and fit less perfectly with its complement to form an energy masker. Viewed another way, the temporal integration function reflects the time over which a contour develops and becomes independent of subsequent 
contours. In this view, the $50 \mathrm{msec}$ or so after the onset of a cutaneous pattern is a period of some "fragility," when additional contours can interfere with the perception of the existing contours. The importance of stimulus onset (rather than stimulus duration or ISI) will be considered below.

The view of the temporal integration function as a measure of both the ability of two patterns to add and the way in which one pattern interferes with another is also a recognition of different task demands. If identification of a pattern requires information from two temporally discrete patterns, then the decline in performance with SOA reflects the inability of the system to integrate. If the first of two patterns is to be identified, then the improvement in performance (decline in masking) is a measure of the emergence of the first pattern. One could imagine designing pairs of stimuli such that on one set of trials the subject is required to integrate the two members of the pair to identify the pattern, and on another set of trials the subject is required to identify only one member of the pair. Performance on the two sets should be mirror images of one another.

The importance of onset has been stressed in the discussion of the results of Experiments 1 and 2. The importance of onset for visual stimuli has been emphasized by Di Lollo. In a series of experiments on temporal integration, Di Lollo (1980) found that, when the leading edge of the first of two patterns was greater than about $100 \mathrm{msec}$, performance requiring integration of the two patterns was greatly impaired. The results indicated that, as in the present case, the ISI (the time between offset of the first pattern and the onset of the second) is not the critical variable in determining the amount of temporal integration. In the case of visual stimuli, such a result is inconsistent with iconic storage hypotheses that maintain that visual persistence declines as a function of the time from the offset, not the onset, of a visual stimulus. The results of Experiments 1 and 2 support a view of tactile storage similar to DiLollo's view.

An additional finding with tactile stimuli is that, in a task that measures integration, performance declines even when stimuli overlap temporally. This is notable because the naive assumption might be that stimuli that temporally overlap would be more likely to integrate than stimuli that do not overlap. Again, the onset of the stimulus appears to be the crucial factor, as both Figures 3 and 4 indicate.

The data presented in Figure 6 also show the critical importance of onset, even when the pattern and complement masks overlap. For the 13-msec maskers, at a 17-msec SOA, for example, the change in masking is $7 \%$ and $10 \%$ for the two sets of measurements. In that condition, there is a 4-msec gap between the offset of the pattern and the onset of the complement.
For the 26-msec maskers at a nearby SOA, $13 \mathrm{msec}$, the change in masking is $7 \%$ and $9 \%$, even though in this condition the pattern mask and complement have no gap between them, but overlap one another by $13 \mathrm{msec}$. These observations suggest that, even as the pattern remains on, the representation of its contours begins to fade.

In previous studies testing modes of pattern generation with the Optacon, pattern elements were usually generated on their own particular area of the fingertip. And, when the onsets of these elements are presented close enough together in time, they can be integrated, producing performance similar to that achieved with a static presentation. At longer SOAs, integration is not possible, and subjects shift from parallel to serial processing. Only in the scan mode, as the Optacon is used as a reading aid, is the entire pattern moved across the array. Thus, when patterns move across the array rapidly, elements strike the same area of skin sequentially and are presumably integrated; however, integration in this case should interfere with recognition. The prediction is that performance should be very poor for the scan mode at the briefest display times, and it is (Craig, 1981).

The presumed effect of temporal integration on modes of pattern generation leads to a prediction about two additional modes, stationary slit and moving slit, both tested on the TVSS (Loomis, 1974). In the former mode, the letter is exposed as though passing behind a stationary, vertical slit. In the latter mode, the pattern is revealed as though the letter was stationary and the slit was moving across it. Using the TVSS, subjects performed about equally well with the two modes. Such a result would be predicted with the large areas of skin and long display times used with the TVSS; subjects should process the pattern serially and not use the additional spatial information provided by the moving slit patterns. With briefer display times, it would be expected that patterns presented in the stationary-slit mode would be very difficult to recognize because, as with the scan mode, the contours should be integrated with one another. The moving slit should suffer relatively less as display time is decreased because processing can change from serial to parallel. Of all the modes tested on the Optacon, the moving slit (referred to as "slit scan," Craig, 1981) mode showed the smallest decline in performance as display times were shortened (Craig, 1981). In the 1981 study, no measurements were made with the stationary slit mode; however, subsequently we compared the two modes at brief ( $4 \mathrm{msec}$ per element) and long (100 msec per element) display times. The results were as predicted by the integration results. With the longer display times, both the stationary and moving slit modes did reasonably well, $37 \%$ and $43 \%$, with the latter showing only a modest decline in performance to $38 \%$ correct as display time was 
reduced. However, as display time was reduced, with the stationary slit, performance suffered considerably more, dropping to $20 \%$ correct.

The importance of onset may also be examined by manipulating the way in which a vibrotactile pattern is turned on. Might it be possible to "ramp on" a pattern so slowly that the pattern cannot be identified? We investigated this possibility by dividing letters into six segments and then generating the letter by turning on and leaving on one of the six segments, then the next, and so forth. Each segment was created by sampling randomly, without replacement, one-sixth of the total pins constituting the letter. The idea was to make identification of the letter difficult, if not impossible, based on the perception of a single segment. In one case, the "ramp time," the time from onset of the first of the segments to the moment when all six segments were present, was $26 \mathrm{msec}$, brief enough to permit the six segments to be integrated. In a second case, the ramp time was $312 \mathrm{msec}$, exceeding the limit over which temporal integration should permit a unified percept. At the end of the ramp time in both cases, all segments of the letter remained on for $52 \mathrm{msec}$. The result was not exactly as predicted, that is, chance performance for the longer ramp, but performance was clearly in the direction predicted, $16 \%$ poorer performance with the longer ramp stimuli than with the shorter. Additional, more direct ways of ramping on patterns, for example, by intensity modulation, are currently being explored.

Of the number of questions the present results raise, one that is of particular theoretical interest is whether the temporal integration functions obtained represent a single function or are the result of the combination of several different temporal integration functions. For example, in combining letter halves, is there one time course for integrating location information on the fingertip and a separate time course for defining a "gap"? Are there different integration functions for generating different tactile features? The task in Experiment 3 yielded the sharpest integration functions and is the "easiest" task, that is, had the fewest response alternatives and the highest overall level of performance. It may be that the critical feature (or features) required to discriminate between $O$ and $X$ has an integration function different from that seen with more difficult tasks; or it may be that "easier" tasks produce sharper functions. Future work will examine temporal integration functions for a variety of features, such as angularity, gaps, line bisection, and so forth, and for several levels of difficulty for each feature. Such measurements should show the extent to which temporal integration functions can be manipulated and may indicate whether such functions are the result of the combination of separable integration functions.

\section{REFERENCES}

Berglund, B., Berglund, U., \& Ekman, G. Temporal integration of vibrotactile stimulation. Perceptual and Motor Skills, $1967,25,549-560$.

BreitmeYer, B. G., \& GANZ, L. Implications of sustained and transient channels for theories of visual pattern masking, saccadic suppression, and information processing. Psychological Review, 1976, 83, 1-36.

CraIG, J. C. Vibrotactile pattern recognition and masking. In G. Gordon (Ed.), Active touch-the mechanism of recognition of objects by manipulation: $A$ multi-disciplinary approach. Oxford: Pergamon Press, 1978.

Craig, J. C. A confusion matrix for tactually presented letters. Perception \& Psychophysics, 1979, 26, 409-411.

Craig, J. C. Modes of vibrotactile pattern perception. Journal of Experimental Psychology: Human Perception and Performance, $1980,6,151-166$.

Craig, J. C. Tactile letter recognition: Pattern duration and modes of pattern generation. Perception \& Psychophysics, 1981, $30,540-546$.

Craig, J. C. Vibrotactile masking: A comparison of energy and pattern maskers. Perception \& Psychophysics, 1982, 31, 523-529.

Di LoLlo, V. Temporal integration in visual memory. Journal of Experimental Psychology: General, 1980, 109, 75-97.

ERIKSEN, C. W., \& Collins, J. F. Some temporal characteristics of visual pattern perception. Journal of Experimental Psychology, 1967, 74, 476-484.

Felsten, G., \& Wasserman, G. S. Visual masking: Mechanisms and theories. Psychological Bulletin, 1980, 88, 329-353.

Gescheider, G. A. Evidence in support of the duplex theory of mechanoreception. Sensory Processes, 1976, 1, 68-76.

Green, B. G. Vibrotactile temporal summation: Effects of frequency. Sensory Processes, 1976, 1, 138-149.

Loomis, J. M. Tactile letter recognition under different modes of stimulus presentation. Perception \& Psychophysics, 1974, 16, 401-408.

Massaro, D. W. Experimental psychology and information processing. Chicago: Rand McNally, 1975.

Turvey, M. T. On peripheral and central processes in vision: Inferences from an information-processing analysis of masking with patterned stimuli. Psychological Review, 1973, 80, 1-52.

VERRILLO, R. T. Temporal summation in vibrotactile sensitivity. Journal of the Acoustical Society of America, 1965, 37, 843-846.

(Manuscript received March 17, 1982; revision accepted for publication June 21, 1982.) 